\title{
El desierto: de la evocación literaria al referente espacial
}

\section{Montserrat Cots Vicente \\ Universitat Pompeu Fabra}

Resumen: El desierto, como referente espacial narratológico, se configura novedosamente en las obras de algunos novelistas del siglo XX: Loti, Saint-Exupéry, Buzzati y Le Clézio entre otros. Dicho espacio desarrolla virtualidades inéditas, conservando al mismo tiempo las características propias del locus horridus.

Palabras clave: desierto, referente espacial

\begin{abstract}
The desert, as spatial narratological reference, is newly shaped in the works of some twentiethcentury novelists: Loti, Saint-Exupéry, Buzzati and Le Clézio, among others. This space develops unprecedented virtualities while maintaining at the same time the specific characteristics of the locus horridus.
\end{abstract}

Keywords: desert, spatial reference

La etimología, a cuya ayuda acudimos hoy escasamente, atestigua que el étimo de "desierto" se encuentra en el verbo latino "deserere" que significaba "abandonar"; la palabra llevaba implícito pues, en su significado primigenio, una connotación negativa. La célebre definición que de este espacio natural hizo el botanista francés dieciochista conde de Buffon en su Histoire naturelle se dirigía en primer lugar a nuestra imaginación, como se 
deduce de la fórmula inicial desiderativa "qu'on se figure", y tenía una evidente intención de sobrecoger al lector con la inclusión de varios rasgos negativos:

Qu'on se figure un pays sans verdure et sans eau, un soleil brûlant, un ciel toujours sec, des plaines sablonneuses, des montagnes encore plus arides, sur lesquelles l'œil s'étend et le regard se perd sans pouvoir s'arrêter sur aucun objet vivant; une terre morte et, pour ainsi dire, écorchée par les vents, laquelle ne présente que des ossements, des cailloux jonchés, des rochers debout ou renversés, un désert entièrement découvert, où le voyageur n'a jamais respiré sous l'ombrage, où rien ne l'accompagne, rien ne lui rappelle la Nature vivante: solitude absolue, mille fois plus affreuse que celle des forêts ; car les arbres sont encore des êtres pour l'homme qui se voit seul ; plus dénué, plus perdu dans ces lieux vuides. ${ }^{1}$

Idéntica visión negativa se había generado a través de ciertos pasajes bíblicos: así el desierto, en tanto que espacio de la nada, imperaba en el cosmos antes de la creación: "El día en que hizo Yahveh Dios la tierra y los cielos, no había aún en la tierra arbusto alguno del campo, y ninguna hierba del campo había germinado todavía" (Génesis 2,11). Precisamente por su vacuidad, el desierto pudo devenir espacio ascético: Jesús vive en él para sufrir las tentaciones del diablo (Evangelios de San Mateo 4,10; de San Marcos 1,12-13; de San Lucas 4,1-13).² Y los anacoretas buscan en el espacio desértico una vía privilegiada de acercamiento a Dios a través de la ascesis. Espacio de reunión de contrarios, "le désert en effet, s'oppose à la terre cultivée. Il est en réalité une terre fertile à l'origine que les malédictions, les dévastations, les guerres, les mauvaises actions de l'homme ont désolée."3 A pesar de ello, en el Salmo 65, Reconocimiento de la bondad de Dios, se exulta la fertilidad del desierto: "rebosan los pastos del desierto/ y las colinas se ciñen de alegría" $(65,13)$.

No es de extrañar que experiencias y visiones tan opuestas hayan hecho del desierto un topos literario. Autores de todas las épocas y de diferentes culturas lo adoptaron como eje espacial narrativo y los poetas como objeto de deseo. Así se han publicado antologías de textos ${ }^{4}$ y simposios científicos ${ }^{5}$ consagrados a demostrar su vitalidad literaria y su interés para las ciencias humanas. Ciertos espacios o lugares, especialmente aquellos que configuran un paisaje, resultan elementos claves en el mundo de la ficción por sus "valores 
añadidos": el desierto, a sus particulares rasgos geográficos, une las expectativas ficcionales que despierta en el lector ya sea por su inmensidad, o por su carácter a menudo terrible e inquietante o incluso, por su ancestral trascendencia religiosa.

Esta capacidad de sugestión puede justificar que presente un acercamiento a dicho espacio a partir de un corpus narrativo que, aunque discutible, presenta en cada caso una singularidad que lo hace interesante para una aproximación geocrítica. Dicho análisis lo inicio con Le désert de Pierre Loti (1895), obra en la que la experiencia del viaje es esencial, para continuar con Terre des hommes de Saint-Exupéry en 1939, fruto de una experiencia humana hasta cierto punto traumática, para adentrarnos en los valores simbólicos que puede alcanzar este espacio en los casos de Il Deserto dei Tartari de Dino Buzzati de 1949 y en el de Désert de Le Clézio en 1980.

Se puede cuestionar, y así se ha hecho, que las obras de ficción sean un punto de apoyo fiable como base para un estudio objetivo de un espacio determinado. Por esta razón vamos a exponer algunos de los presupuestos adoptados a partir de la perspectiva teórica de la geocrítica que propugna Bertrand Westphal6: para este autor, cualquier espacio geográfico usado en la ficción puede adoptarse como "référent acceptable" (p. 186); a partir de esta premisa, pueden establecerse relaciones entre los diversos escritores que recurran a él, aún teniendo en cuenta sus representaciones individuales; nace así una "dynamique multifocale" (p. 191) que no puede más que enriquecer la aproximación al estudio; al mismo tiempo esta realidad ficcional tiene capacidad para influir sobre lo real y da como resultado "un réel élargi" (p. 191) que redundará en beneficio del estudio del espacio geográfico. En esta misma dirección, más matizada narratológicamente, se tienen en cuenta las opiniones de Françoise Lavocat para quien, en la ficción, son precisamente las verdades no referenciales las que desencadenan inferencias que son para los lectores elementos de verdad, de tal modo que: "En effet, les propositions fictionnelles construisent aussi leur propre domaine de référence, ce qui estle fondement de leur valeur assertive".7 De manera más general, Juan José Saer añade a estos planteamientos que: "El rechazo escrupuloso de todo elementos ficticio no es un criterio de verdad." 8

La ficción contiene pues en sí misma la posibilidad de crear un mundo posible a 
través de un espacio determinado y el lector puede reconstruir este o estos mundo(s) en el ejercicio de su capacidad lectora, ya sea en la ficción o también en el relato de viaje, en cuanto a la dimensión subjetiva que este último género encierra.

Esta dimensión subjetiva de la que se desprende una visión personal se hace patente en Le désert, el relato del viaje que Pierre Loti emprendió en 1894 para visitar el monasterio de Santa Catalina y la ciudad de Petra - cosa esta última que no logró - . Se puede observar en el texto la inquietud religiosa del autor que incide en la visión del paisaje: confiesa que le ha movido su deseo de ver "la sainte Jérusalem" y que por ello escoge una ruta determinada ya que desea "y venir par les vieilles routes abandonnées et préparer mon esprit dans le long recueillement des solitudes" (p. 2). La ruta escogida es, sin embargo, la más larga y resulta impracticable: se trata de atravesar el Sinaí, llegando a Akabah y penetrar finalmente en el desierto de Petra para visitar las ruinas arqueológicas; dicha aspiración no se realizará porque no obtendrá el permiso necesario. El narrador observa, ya en el camino, que se halla ante un "infini vide", descripción con ecos pascalianos ${ }^{9}$, y la experiencia vivencial que le genera es "une sorte d'ivresse et de frisson de solitude. “ El lenguaje empleado en la descripción del paisaje es más sugestivo que descriptivo aunque transmite a la perfección la inquietud del sujeto.

Desde el inicio del viaje, emerge la visión negativa del narrador. Unas pinceladas descriptivas herederas de la descripción del locus horridus configuran la pintura del desierto: el adjetivo "sinistre" se repite a menudo: "vallées sinistres" (p. 29), "comme elle est silencieuse, sinistre et froide cette apparition de la montagne" (p. 42), "il fait lourdement chaud, et surtout il fait sinistre” (p. 175), “ les sinistres montagnes du pays d’Edom ” (p. 183), "toutes ces montagnes... prennent de sinistres couleurs" (p. 214); también aparecen pinceladas paisajísticas metaforizadas que sugieren miedo o inquietud: "rochers de cauchemar" (p. 39), "puis vient la nuit (...) Et c'est à ce moment, une impression d'effroi presque religieux." (p. 26). El espanto se expande también por el miedo a ser robados, lo que acrecienta la impresión de inseguridad de la caravana.

Sin embargo, no todo es negativo en esta experiencia vivencial del desierto: desde el punto de vista estético, Pierre Loti se muestra extremadamente sensible al color, a los 
cambios lumínicos, así como a la capacidad de metamorfosis colorativa de este espacio: "voici l'heure du couchant, l'heure du magique" (p. 21); "Nous sommes dans un pays tout rose, marbré de bleu pâle” (p. 107), “L’Arabie déserte (...) déroule l'infini de ses désolations roses" (p. 183). Pero esta sinfonía de color se contagia a veces de la negatividad del desierto y se convierte también entonces en "sinistre" (p. 214).

Cuando el viaje finaliza y entran en Palestina es un Viernes santo; el pensamiento del narrador va hacia el Crucificado y resume la experiencia de su viaje con una consideración sobre el desierto como espacio de la inmensidad que permite al hombre considerarse un rey: “Au désert, on était des rois disposant d'espaces sans mesure” (p. 239). La observación de Loti sobre el espacio inconmensurable del desierto nos remite a la distinción que estableció su contemporáneo Henri Bergson entre espacio y “étendue": para el filósofo, es la "étendue" la que atrae nuestra percepción: "Ce qui est perçu, c'est l'étendue colorée, résistante". 10 Y es esta amplitud intrínseca que sobrecoge y deja honda huella en el ser humano. Loti, aunque viajero infatigable, se muestra sensible a las sensaciones que despierta la magnitud de un desierto.

Las reacciones de Loti ante el desierto son distintas a las de Antoine de SaintExupéry. Roger Caillois hizo de este autor un breve retrato devenido célebre: "SaintExupéry n'est pas essentiellement un homme de lettres, en particulier par la conception qu'il a de la littérature. Il est un homme d'action à qui l'action ne suffit pas "11 El hombre de acción lo encontramos reflejado sin duda en su faceta de piloto. Saint-Exupéry, piloto de correo postal en sus inicios, piloto profesional a partir de 1933, piloto de guerra a partir de 1939, ofrece una visión del desierto filtrada a través del conocimiento real y de las experiencias vividas a través de su propia reflexión. Pero sin duda, el rasgo que define su experiencia con este espacio es la toma de conciencia del valor de la perspectiva aérea en la visión y relación con el paisaje: "L'avion est une machine sans doute, mais quel instrument d'analyse! Cet instrument nous a fait découvrir le vrai visage de la terre. Les routes, en effet, durant des siècles, nous ont trompés" (p. 171). ${ }^{12}$ Y en esa misma dirección observa: "Mais un autre miracle de l'avion est qu'il vous plonge directement au cœur du mystère" (p. 180).

Además de la perspectiva aérea desde la que contempla el desierto, Saint-Exupéry 
es consciente del riesgo posible y de la muerte latente que dicho espacio encierra; ello se refleja en una de sus obras más carismáticas, Terre des Hommes, publicada en 1939, la cual, según Michel Quesnel "n’a rien d’un roman"13, opinión reformulada por Maria de Jesus Cabral quien recuerda que su composición reúne diversas experiencias vividas y que por ello la obra se caracteriza "par son hybridisme narratif et discursif”, ${ }^{14}$ mezcla de relatos, recuerdos y reflexiones. Entre los relatos vivenciales está el del accidente de su avión en el desierto libio, junto con Prévot, espacio del cual describe su grado de humedad en tanto que buen "connaisseur": "Il me revient à la mémoire ce que je sais du désert de Lybie. Il subsiste, dans le Sahara, 40 pour cent d'humidité, quand elle tombe ici à 18 pour cent. Et la vie s'évapore comme une vapeur" (p. 225). Atento al entorno, cuando el avión se ha estrellado, observa las huellas de un tipo de zorros o "fénechs", "petits carnivores gros comme des lapins et ornés d'énormes oreilles"15 (p. 226). También se fija atentamente en cómo estos animales se aprovisionan de pocos caracoles en los arbustos, con la precaución de preservarlos para el futuro, ejemplo de adaptación al medio natural del cual el piloto saca una provechosa lección de supervivencia.

La descripción de los kilómetros recorridos en busca de ayuda, después del accidente, sin agua, bajo un sol de justicia, sufriendo numerosos espejismos, vanas esperanzas fruto del engaño de los sentidos, confieren al desierto un poder temible, letal para el ser humano: “Et je découvre que dans le désert il n'est point de refuge. [...] Il ne forme point d'ombre pendant le jour, et la nuit il vous livre tout nu au vent. Pas un arbre, pas une haie, pas une pierre qui m'eût abrité" (p. 236).

Sin embargo, frente a esta vivencia terrible, se erige el recuerdo en el pasado del Sahara como contrapunto positivo: "J'ai beaucoup aimé le Sahara. J'ai passé des nuits en dissidence. Je me suis réveillé dans cette étendue blonde” (p. 220). E incluso se produce una curiosa simbiosis entre el sujeto Saint-Exupéry y el espacio del desierto; en el inicio del capítulo sexto el autor reconoce que el desierto ha sido para él una escuela de soledad; y ello porque es un espacio propicio a la meditación, pero también un espacio que permite a nuestro autor llevar a cabo una toma de conciencia sobre su propia existencia y su condición humana: "Et je méditai sur ma condition, perdu dans le désert et menacé, nu 
entre le sable et les étoiles, éloigné des pôles de ma vie par trop de silence. [...]. Je n'étais rien qu'un mortel égaré entre du sable et des étoiles, conscient de la seule douceur de respirer." (p. 177)

La visión idealizada e idílica del desierto en algunos momentos no impide sin embargo que éste conserve su carácter maléfico, asesino, especialmente cuando el reducido equipo del avión se ve aprisionado en él. La pregunta sin respuesta: "combien de temps résisterons-nous?“ (p. 168) expresa la inquietud de su ánimo: como en la tragedia griega, la maldición puede ser inminente pero el autor-narrador asume su suerte sin pestañear, llevando a la culminación su condición de héroe: "Je ne regrette rien. J'ai joué, j’ai perdu" (199). A pesar de las evocaciones líricas citadas, el desierto conserva su poder terrible que ejerce sobre la criatura humana de forma inexorable.

En Terre des hommes, Saint-Exupéry marca un antes y un después en la evocación literaria del desierto por el carácter vivencial que transmite su conocimiento de este espacio: no es un espacio simbólico sino un espacio de la experiencia vivida, que puede resultar letal para el viajero o el piloto que se pierde y que ha de permanecer en él bajo un sol abrasador. La visión del desierto que nos transmite Saint-Exupéry vehicula una visión del desierto como espacio épico en el cual sólo sobreviven aquellos hombres de gran capacidad física y resistencia moral.

Un año después de Terrre des Hommes Dino Buzzati publicó en Milán su obra maestra, Il deserto dei Tartari. A pesar de la relevancia que el título parece conceder al desierto, dicho espacio ha perdido su carácter terrible para el ser humano. La novela de Buzzati transcurre, de facto, en el espacio limitado de la Fortaleza Bastiani, a la cual llega un joven oficial, Giovanni Drogo, con la ilusión de hacer una brillante carrera militar, ilusión que, desde el inicio, se contrapone a una realidad espacial anodina, sin un atisbo de dimensión épica:

- "È un tratto di frontiera morta" aggiunse Ortiz. [...]

- “Come: frontiera morta?"

- “Una frontiera che non dà pensiero. Davanti c'è un grande deserto".

- “Un deserto? 
- “Un deserto effettivamente, pietre e terra secca, lo chiamano il deserto dei Tartari." (p. 18)

Como resume el Mayor Monti “è un paesaggio stupidissimo" (p. 30), exento del carácter terrible o pavoroso del desierto. En la obra de Buzzati, el desierto pierde la aureola de espacio de la aventura o la dimensión de lo épico. A la insignificancia del espacio, reducido de hecho a la fortaleza, se añade la coordenada del tiempo, con su transcurrir monótono, que acabará paulatinamente con las esperanzas de una ansiada batalla; el temor a los tártaros pierde consistencia y la realidad, banal y monótona, se impone: "il deserto risplandeva più solitario che mai” (p. 91). Así, los soldados se ven forzados a admitir que “Oramai la strada è abbandonata, dal nord non verrà più nessuno” (p. 196). La posibilidad de un ataque se reduce con el transcurrir del tiempo; también para Drogo, convertido en un soldado enfermo y moribundo que debe abandonar la fortaleza, precisamente cuando el peligro de la llegada de soldados enemigos se dibuja como posible.

A lo largo de la novela, se percibe que el peligro ha de venir del desierto, o mejor de: "una specie di deserto, lastricato di rocce [...] desolata pianura, priva di senso e misteriosa" (p. 83). El desierto no se configura como tal por la locución restrictiva que le antecede "specie di"; por ello los soldados han de suplir con la imaginación visiones fantasmagóricas de tártaros avanzando por el desierto: “Qualcosa si avvicinava dal settentrione, come mai era successo a memoria di uomo. Aumentando la luce, sul fondo bianco del deserto spiccò nitida la schiera umana che avanzava" (p. 104). Los soldados de la Fortaleza Bastiani ven enemigos imaginarios en la "pianura" de los Tártaros, aunque finalmente exista un ataque real y para Giovanni Drogo sea ya demasiado tarde.

Como en Terre des hommes, el desierto mantiene en la novela de Buzzati su capacidad de despertar ensoñaciones heroicas, aunque éstas resulten fallidas. La perspectiva de la obra es pesimista: el desierto, como espacio en sí mismo, no se describe pero sí se asimila a una frontera (la sinonimia en la designación vacila entre "deserto" y "pianura") de la cual proviene el peligro, el peligro de los Tártaros o de cualquier otro enemigo. Entre tanto, la vida de un hombre, el soldado Drogo, se desvanece.

Numerosos críticos han coincidido en que Jean-Marie Gustave Le Clézio es hoy uno de los mayores referentes literarios que muestran una preocupación manifiesta ante las 
agresiones humanas a la Naturaleza. ${ }^{16}$ Según se desprende de sus propias palabras, no hay espacios teóricamente inhabitables que le resulten repulsivos o peligrosos, entre ellos, el desierto; así lo manifestaba en una entrevista a Bernard Pivot en "Apostrophes" en la cual sostenía que los lugares inhóspitos no dejan por ello de ser la cuna de grandes civilizaciones, opinión que también se recoge en Gens de nuages: "Les grandes civilisations qui ont éclairé le monde ne sont pas nées au paradis. Elles sont apparues dans les régions les plus inhospitalières de la planète, sous les climats les plus difficiles. Dans les déserts brûlants de l'Irak, en Anatolie, en Judée, en Égypte, au Soudan."17

Sin lugar a dudas es en la novela Désert, publicada en 1980, donde Le Clézio expresa su devoción manifiesta por este espacio, comenzando por el título en el que tenemos "un nom commun élevé, avec sa majuscule, l'absence d'article, à la possible dignité de nom propre." 18En efecto, el desierto concentra la doble trama que preside la obra: la hazaña histórica de Ma El Aïnin cuando convoca a los hombres azules a la guerra contra los colonizadores y la historia de Lalla, la joven que encuentra en el desierto el amor de Le Hartani y la felicidad que no le ofrece la ciudad; ésta, representada por Marseille, se ha convertido en un reducto para desheredados, más terrible que el desierto mismo: “ici c'est la peur du vide, de la détresse, de la faim, la peur qui n'a pas de nom" (p. 262).

Las coordenadas geográficas de la novela están bien identificadas y se centran en la región desértica de la Saguia el Hamra, en Marruecos, con una geografía específica, descrita también en Gens des nuages: “une vallée asséchée à l'extrême sud du Maroc, au-delà du Draa, au coeur d'un territoire qui a longtemps appartenu à l'Es pagne sous le nom de Rio de Oro" (p. 15). Dos observaciones críticas son interesantes para nuestro estudio: en primer lugar la de Madeleine Borgomano quien observa que en la producción de Le Clézio “chez cet amateur de réel, c'est toujours le roman qui précède le récit de voyage"19 refiriéndose en concreto a Désert frente a Gens des nuages y a Le Chercheur d'or frente a Voyage à Rodrigues. Y en la misma dirección Chrystele Sohy subraya que "l'auteur a écrit sur une région marocaine, avant même d'en avoir la connaissance réelle."20

¿Afecta esta falta de conocimiento previo y real a la referencialidad de la descripción? El lector de la obra observa que la descripción del paisaje se mantiene 
voluntariamente indeterminada, imprecisa, acudiendo a menudo a epítetos más sugestivos que denotativos, privilegiando la descripción evocadora a la descripción geográfica : "plaines de roches coupantes, montagnes déchirantes, crevasses, nappes de sable qui réverbéraient le soleil " ( p.21); "Le désert est comme la mer, avec les vagues du vent sur le sable dur, avec l'écume de broussailles roulantes, avec les pierres plates " (p. 169) ; "le désert qui rutile et ondoie, les gerbes d'étincelles, les lentes vagues des dunes qui avancent vers l'inconnu" (p. 190). El carácter impreciso de la descripción contrasta con la precisión de los topónimos en la descripción geográfica, ya que además del valle de Saguiet el Hamra se citan otros lugares frecuentados por los hombres del desierto: "les routes qui viennent du Draa, de Tamgrout, de l'Erg Iguidi, ou, plus au nord, la route des Ait Atta, des Gheris, de Tafilelt qui rejoignent les grands ksours des contreforts de l'Atlas " (p. 12); y se señalan sus diversas rutas como si la información proviniera de un atlas: "Ils étaient venus de tous les points du désert, au-delà de la Hamada de pierres, des montagnes du Cheheïba et de Ouarkzi , du Siroua, des monts Oum Chakourt, au-delà même des grandes oasis du Sud, du lac souterrain de Gourara" (p. 14). También en este caso se evidencia que la descripción geográfica está bien documentada.

Otros dos elementos configuran el espacio del desierto en la obra de Le Clézio: la luz y el viento. La luz tiene una importancia capital; si el epíteto que la acompaña a menudo es "belle, "21 resulta sin embargo maléfica para el hombre porque le deslumbra y quema sus ojos: "On vivait, on mourait, toujours en regardant avec des yeux fixes brûlés de fatigue et de lumière" (p. 19); la quemadura de la luz es además seca y polvorienta, en consonancia con el espacio en que se halla: "La brûlure de la lumière est sèche et poudreuse. " (p. 201). Lalla experimenta sus terribles efectos en el momento de dar a luz porque tiene "le visage brûlé par la splendeur de la lumière", en una poética oposición entre la belleza de la luz (splendeur) y su poder nocivo (brûlé).

Al igual que la luz, el viento también resulta nefasto: se podría decir que, contrariamente a lo esperado en buena lógica ya que es portador de frescor o de brisa, el viento levanta la arena ardiente: "Le vent du désert soufflait maintenant par intermittence, jetant au visage des hommes des grains de sable qui brûlaient la peau" (p. 53); o, cuando 
sopla, seca los pocos paisajes acogedores que se encuentran: " mais la palmeraie était petite, rongée par la sécheresse et par le vent du désert" (p. 230). Observamos pues que, en el desierto de Le Clézio, elementos de la naturaleza que no son nocivos ontológicamente, lo devienen en dicho entorno natural. Por el contrario, el desierto puede encerrar cualidades humanas privilegiadas como el silencio. Recordemos que para George Steiner "hay acciones del espíritu enraizadas en el silencio," 22 y el autor nos recuerda su valor en la vida monástica y especialmente para los Padres del desierto. Desde las primeras páginas de la novela, Le Clézio otorga a los Hombres Azules la capacidad del silencio: "Ils ne disaient rien [...] La sécheresse avait durci leurs lèvres et leur langue [...] muets comme le désert" (p. 8). El silencio se confunde y se asimila con el desierto y por ello deviene un elemento bienhechor para Lalla en una de sus desoladoras noches en Marsella: "Lalla marche le long des quais, vers la mer. Ici, tout d'un coup, c'est le silence, comme si elle était vraiment arrivée dans le désert" (p. 275).

Magri-Mourgues ${ }^{23}$ observa que otro rasgo característico de la escritura del desierto es la descripción negativa para evocar el infinito espacial: "les pierres et le sable sans fin", "l'étendue sans fin de la pierre et de la poussière". Ese infinito espacial es un rasgo común del desierto y del mar, espacios del "no límite" que encuentran su esencia en una inmensidad negativa: a partir de la idea del "no límite" o, dicho de otra forma, cuando el "limes" se supera, ${ }^{24}$ puede expresarse una poética del desierto como encontramos en la novela de Le Clézio.

La descripción del desierto en este autor va más allá de las acotaciones geográficas o de la tópica descriptiva ad usum. Así el desierto simboliza valores épicos, que habíamos ya encontrado en Saint-Exupéry, aunque con un registro distinto: la realidad histórica da fe de la rebelión dirigida por Ma el Aïnine contra los franceses y de su derrota definitiva: "Sa Harka est dispersée par les troupes du général Moinier (23 juin 1910)."25 Le Clézio posa su mirada cómplice sobre aquellos hombres andrajosos, "sans armes, qui courent en boîtant" (p. 360), aquellos "hommes bleus" que corren "sans but, sans fin" sobre la tierra seca y así confiere dignidad a su derrota. En el polo opuesto a la muerte o a la negación de vida, en el desierto también se accede al encuentro de los amantes y a la fusión de sus cuerpos: Lalla y 
Le Hartani viven su noche de amor bajo las estrellas, "et c'est un très grand vertige qui les traverse, qui agrandit leurs pupilles" (p. 205). El desierto es también paraíso de amor y de plenitud humana. Como otros espacios, la montaña por ejemplo, también el desierto bascula entre locus horridus y locus almus.

Bertrand Westphal nos recuerda que las relaciones existentes entre literatura y espacios humanos son dinámicas y que además, interfieren entre ellas: “L'espace transposé en littérature influe sur la représentation de l'espace dit réel (référentiel), sur cet espacesouche dont il activera certaines virtualités ignorées jusque-là, ou réorientera la lecture."26 En efecto, el desierto forma parte de un topos espacial establecido que, de manera más o menos ostentosa, estará presente en cualquier obra que integre este espacio y que comprenderá sol abrasador, dunas, arena, falta de agua, vacío, silencio... ; sin embargo, a partir de esta tópica, cada autor construirá "su" visión del desierto que exterioriza una determinada opción creativa; así, en Désert, Le Clézio construye, a partir de su particular enfoque, un desierto mítico-simbólico: “c'était le seul, le dernier pays libre” (p. 13), o “c'est le regard de Lalla qui porte la force brûlante du désert" (p. 312). El lector, en su particular relación con el texto, incorporará nuevos elementos que devienen reconocibles y son reconocidos como existentes - aunque solo lo sean en la ficción- en una operación de referencia intraficcional, aquella que permite "construire un monde par le langage" 27 . El estatuto de la ficción así entendido mejorará también sin duda nuestro conocimiento del mundo así como de nuestro imaginario lector. 


\section{NOTAS}

1 Buffon, Georges-Louis Leclerc, comte de; Daubenton, Louis-Jean Marie, Guéneau de Montbeillard, Philibert; Bexon, Gabriel-Léopold-Charles-Aimé, Histoire naturelle, générale et particulière, avec la description du Cabinet du Roi, Paris, Imprimerie Royale, 1749-1789, 36 vols., tome XIe, 1763, p. 220. http://books.google.com

2 Agnès Bastit, en su artículo "Les tentations de Jésus au désert : interprétation patristique", publicado en Le désert, un espace paradoxal, editado por Gérard Nauroy, Pierre Halen y Anne Spica, Bern, Peter Lang, 2003, pp. 79-98, p. 79, hace observar que el evangelio de San Juan no incluye las tentaciones del desierto.

${ }^{3}$ Armand Abécassis, “L'expérience du désert dans la mentalité hébraïque. Le désert de la Genèse ", in

Cahiers de l'Université Saint Jean de Jérusalem, no 8, Le désert et la Queste. Colloque tenu à Paris les 12,13, 14 juin 1981, Paris, Berg International, 1982, pp. 25-43, p. 31.

4 Jacqueline Michel, Jouissance des déserts dans la poésie contemporaine, Paris-Caen, Les Lettres Modernes/Minard, 1998.

5 Gérard Nauroy, Pierre Halen, Anne Spica (eds.), Le désert, espace paradoxal. Actes du colloque de l'Université de Metz (13-15 septembre 2001), Bern, Berlin, Bruxelles, Frankfurt am Main, New York, Oxford, Wien, Peter Lang, 2003.

${ }^{6}$ Westphal, Bertrand. La géocritique. Réel, fiction, espace, Paris, Les Editions de Minuit, 2007.

${ }^{7}$ Lavocat, Françoise. Fait et fiction. Pour une frontière, Paris, Seuil, 2016, p. 388.

8 Saer, Juan José. El concepto de ficción, Barcelona, Editorial Rayo Verde, 2016, p. 14.

9 Pascal, Blaise. “Le silence éternel de ces espaces infinis m’effraie”, Pensées, Paris, Gallimard, 1969, p. 58.

10 Bergson, Henri. L'évolution créatrice, in CEuvres, Paris, Presses Universitaires, 1907, p. 157.

11 Saint-Exupéry, A. de. CEuvres. Préface de Roger Caillois, Paris, Gallimard, 1959, Bibliothèque de La Pléiade, p. XII.

${ }^{12}$ Las citas de las páginas están tomadas de la edición de Saint-Exupéry, A. de. CEuvres. Préface de Roger Caillois, Paris, Gallimard, 1959, Bibliothèque de La Pléiade.

13 Quesnel, Michel. “La création chez Saint-Exupéry ”, Etudes littéraires, vol. 33, no 2, 2001, pp. 13-26, p. 17. DOI: $10.7202 / 501290$ ar

${ }^{14}$ Cabral, Maria de Jesus. "Terre des hommes de Saint-Exupéry : la conquête d'un nouvel espace ", Mathésis 9, 2000, pp. 105-140, p. 106.

N. ${ }^{\circ} 38-6 / 2018$ | 193-209 - ISSN 2183-2242 | http:/dx.doi.org/10.21747/21832242/litcomp38a11 
15 Efectivamente la observación de Saint-Exupéry se ajusta a la realidad: "Le fennec est un petit canidé aux oreilles exceptionnellement larges, joliment bordées de blanc, agrémentées à l'intérieur d'une frange de longs poils [...] ", Bruno Doucey (dir.) Le Livre des Déserts, Paris, Robert Laffont, p. 301.

16 Véanse las citas y referencias aducidas por M. J. Sueza Espejo, "Désert de J.-M. G. Le Clézio: analyse d'éléments descriptifs et interprétation écocritique ", Cédille, no 5, avril, 2009, pp. 329-346.

17 Citado por Gérard de Cortanze, J.-M. G. Le Clézio, Paris, Gallimard, 2009, p. 41.

18 Domange, Simone, Le Clézio ou la quête du désert, Paris, Imago, 1993, p. 25.

19 Borgomano, Madeleine, "Le Clézio ou le voyage dans tous ses états", in Roman et récit de voyage. Textes réunis par Marie-Christine Gomez-Geraud et Philippe Antoine, Presses de l'Université Paris-Sorbonne, 2001, pp. 183-190, p. 184.

${ }^{20}$ Sohy, Chrystelle, “J.-M. G. Le Clézio et le Maroc : Désert, Gens des Nuages", in Christiane Chaulet-Achour (dir), Itinéraires intellectuels entre la France et les rives sud de la Méditerranée, Paris, Karthala, 2010, pp. 47-60, p. 48.

${ }^{21}$ Domange, Simone. Le Clézio ou la quête du désert, Paris, Imago, 1993, p. 51.

22 Steiner, George. Lenguaje y silencio. Ensayos sobre la literatura, el lenguaje y lo inhumano, Barcelona, Gedisa, 2003, p. 29.

23 Magri-Mourgues, Véronique. "La légende du désert : Désert de Le Clézio ", in Pierre Halen, Gérard Nauroy, Anne Spica (éds.), Le désert, un espace paradoxal, Peter Lang, 2003, pp. 467-487, p. 483.

${ }^{24}$ Recordemos la importancia del concepto de "límite" en el conjunto de la obra filosófica de Eugenio Trías, mayormente definido en Los límites del mundo, Barcelona, Ariel, 1985.

25 Désiré-Vuillemin, G.-M. "Cheikh Ma El Aïnin et le Maroc, ou l'échec d'un moderne Almoravide”, Revue d'Histoire des colonies, 1958, vol. 45, no 158, pp. 29-52.

www.persee.fr/doc/outre $0399 \_13851958$ num 451581290.

26 Westphal, Bertrand. "Pour une approche géocritique des textes. Esquisse", in Faculté des Lettres et des Sciences Humaines de Limoges, La géocritique mode d'emploi, Presses Universitaires de Limoges, 2000, pp. 9 39, p. 21.

27 Lavocat, Françoise. Op. Cit., p. 390.

N. 38 - 6/ 2018 | 193-209 - ISSN 2183-2242 | http:/dx.doi.org/10.21747/21832242/litcomp38a11 


\section{Bibliografía}

Abécassis, Armand (1982), “L’expérience du désert dans la mentalité hébraïque. Le désert de la Genèse", in Le désert et la Queste. Colloque tenu à Paris les 12,13, 14 juin 1981, Cahiers de l’Université Saint Jean de Jérusalem, no 8, Paris, Berg International, p. 31, pp. 25-43.

Bastit, Agnès (2003), "Les tentations de Jésus au désert: interprétation patristique”, in Gérard Nauroy, Pierre Halen \& Anne Spica (eds.), Le désert, un espace paradoxal, Bern, Peter Lang, pp. 79-98.

Bergson, Henri (1907), L'évolution créatrice, in OEuvres, Paris, Presses Universitaires.

Borgomano, Madeleine (2001), “Le Clézio ou le voyage dans tous ses états ", in Roman et récit de voyage. Textes réunis par Marie-Christine Gomez-Geraud et Philippe Antoine, Paris, Presses de l’Université Paris-Sorbonne, pp. 183-190.

Buffon, Georges-Louis Leclerc, comte de/ Daubenton, Louis-Jean Marie/ Guéneau de Montbeillard, Philibert / Bexon, Gabriel-Léopold-Charles-Aimé (1749-1789), Histoire naturelle, générale et particulière, avec la description du Cabinet du Roi, Paris, Imprimerie Royale, 1749-1789, 36 vols., tome XIe, 1763.

Buzzati, Dino (1991), Romanzi e racconti, Milano, Arnoldo Mondadori.

Cabral, Maria de Jesus (2000), “Terre des hommes de Saint-Exupéry: la conquête d'un nouvel espace ", Mathésis 9, pp. 105-140.

Cortanze, Gérard de (2009), J.-M. G. Le Clézio, Paris, Gallimard.

Désiré-Vuillemin, G.-M. "Cheikh Ma El Aïnin et le Maroc, ou l'échec d'un moderne Almoravide ", Revue d'Histoire des colonies, 1958, vol. 45, no 158, pp. 29-52.

www.persee.fr/doc/outre 039913851958 num 451581290.

Domange, Simone (1993), Le Clézio ou la quête du désert, Paris, Imago.

N. ${ }^{3} 38-6 / 2018$ | 193-209- ISSN 2183-2242 | http:/dx.doi.org/10.21747/21832242/litcomp38a11 
Doucey, Bruno (dir.) (2006), Le Livre des Déserts. Itinéraires scientifiques, littéraires et spirituels, Paris, Robert Laffont.

Lavocat, François (2016), Fait et fiction. Pour une frontière, Paris, Seuil.

Le Clézio, J.-M. G. (1980), Désert, Paris, Gallimard.

Magri-Mourgues, Véronique (2003), “La légende du désert: Désert de Le Clézio”, in Pierre Halen, Gérard Nauroy/ Anne Spica (éds.), Le désert, un espace paradoxal, Peter Lang, pp. 467-487.

Michel, Jacqueline (1998), Jouissance des déserts dans la poésie contemporaine, Paris-Caen, Les Lettres Modernes/Minard.

Nauroy, Gérard / Halen, Pierre / Spica, Anne (eds.) (2003), Le désert. Un espace paradoxal. Actes du colloque de l’Université de Metz (13-15 septembre 2001), Bern, Peter Lang.

Pascal, Blaise (1969), Pensées, Paris, Gallimard.

Quesnel, Michel (2001), “ La création chez Saint-Exupéry ”, Etudes littéraires, vol. 33, no 2, pp. 13-26. DOI: 10.7202/501290ar

Saer, Juan José (2016), El concepto de ficción, Barcelona, Editorial Rayo Verde.

Saint-Exupéry, A. de (1959), OEuvres. Préface de Roger Caillois, Paris, Gallimard, Bibliothèque de La Pléiade.

Sohy, Chrystelle (2010), “J.-M. G. Le Clézio et le Maroc: Désert, Gens des Nuages", in Christiane Chaulet-Achour (dir), Itinéraires intellectuels entre la France et les rives sud de la Méditerranée, Paris, Karthala, pp. 47-60.

Steiner, George (2003), Lenguaje y silencio. Ensayos sobre la literatura, el lenguaje y lo inhumano, Barcelona, Gedisa.

Sueza Espejo, M. J. (2009), “Désert de J.-M. G. Le Clézio: analyse d’éléments descriptifs et interprétation écocritique ", Cédille, no 5, abril, pp. 329-346.

Westphal, Bertrand (2000), "Pour une approche géocritique des textes. Esquisse ", in 
Faculté des Lettres et des Sciences Humaines de Limoges, La géocritique mode d'emploi, Presses Universitaires de Limoges, pp. 9-39.

-- (2007), La géocritique. Réel, fiction, espace, Paris, Les Editions de Minuit.

Montserrat Cots Vicente es Catedrática de Universidad de Literatura Francesa en la Universidad Pompeu Fabra de Barcelona. Trabaja desde hace años desde presupuestos comparatistas. Há sido Presidenta desde 2008 a 2014 de la Sociedad Española de Literatura General y Comparada y há colaborado en la organización de congrsos internacionales que han culminado en publicaciones como Interrogating Gazes. Comparative Critical Views on the Representation of Foreignness and Otherness, editado, junto con P. Gifra y G. Hambrook, por Peter Lang en 2013. 